\title{
Perinephric abscess following uncomplicated ureteroscopy
}

\author{
Bishoy A. Gayed, MD; Bruce L. Jacobs, MD; Stephen V. Jackman, MD
}

\section{Abstract}

We report the case of a 60-year-old male who presented with left-sided back pain 1 month after an uncomplicated ureteroscopy and stone-basketing for left 6-mm mid-pole and 4-mm lower pole stones.

Can Urol Assoc J 2010;4(3):E74-E75

\section{Case report}

A 60-year-old male presented with left-sided back pain 1 month after an uncomplicated ureteroscopy and stone basketing for left $6 \mathrm{~mm}$ mid-pole and $4 \mathrm{~mm}$ lower pole stones.

Past cultures were negative and the patient was given intravenous levofloxacin preoperatively. Although the patient was not pre-stented, a 12/14 French ureteral access

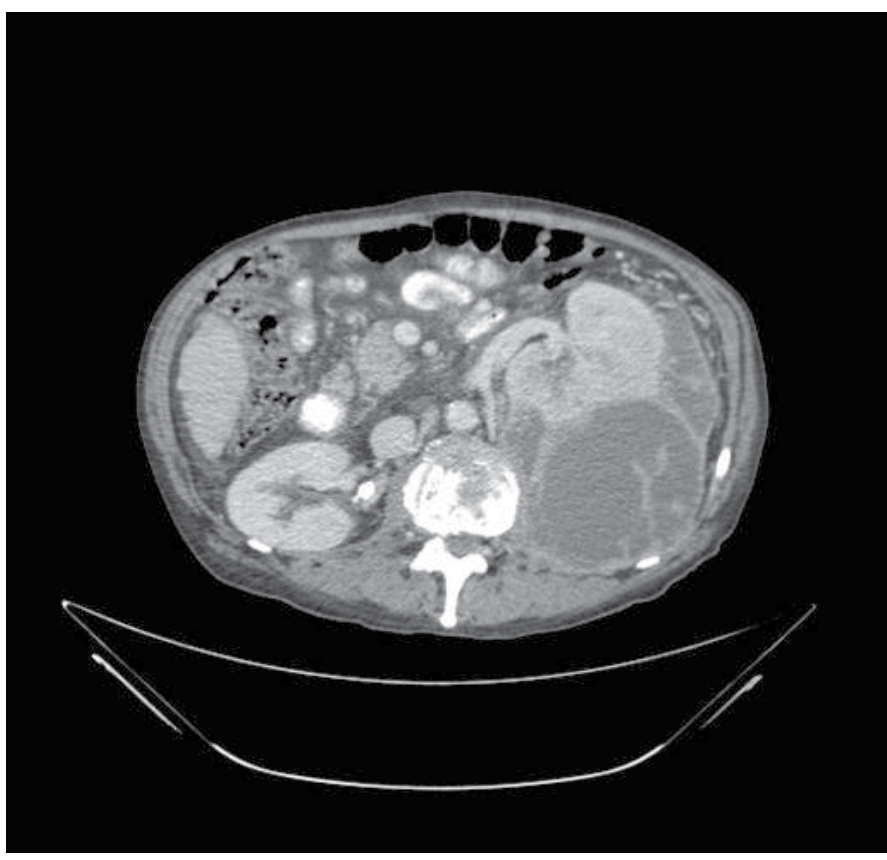

Fig 1a. A computed tomography scan (transverse) on initial presentation. sheath was easily advanced into position under fluoroscopic guidance. A $6 \mathrm{Fr} \times 26 \mathrm{~cm}$ stent, on a tether, was placed at the end of the case and the patient was discharged that day. The clinic stent removal was performed 3 days later.

Our patient presented to the emergency room 1 month postoperatively with left flank tenderness and pain with left leg adduction. He had no fevers or chills. His white blood count was 16000 and creatinine $(\mathrm{Cr}) 1.3 \mathrm{mg} / \mathrm{dL}$ (baseline 1.0). Urine culture at the time of presentation was negative. A large perinephric abscess was seen on computed tomography (Fig. 1a) (Fig. 1b) and a percutaneous drain was placed (Fig. 2). Immediately, 400 cc of purulent fluid was drained. Fluid Cr was $1.0 \mathrm{mg} / \mathrm{dL}$. Cultures grew methicillin-sensitive Staphylococcus aureus, and a prolonged course of nafcillin was started. After 4 weeks, the drain output was minimal and the percutaneous drain was removed.

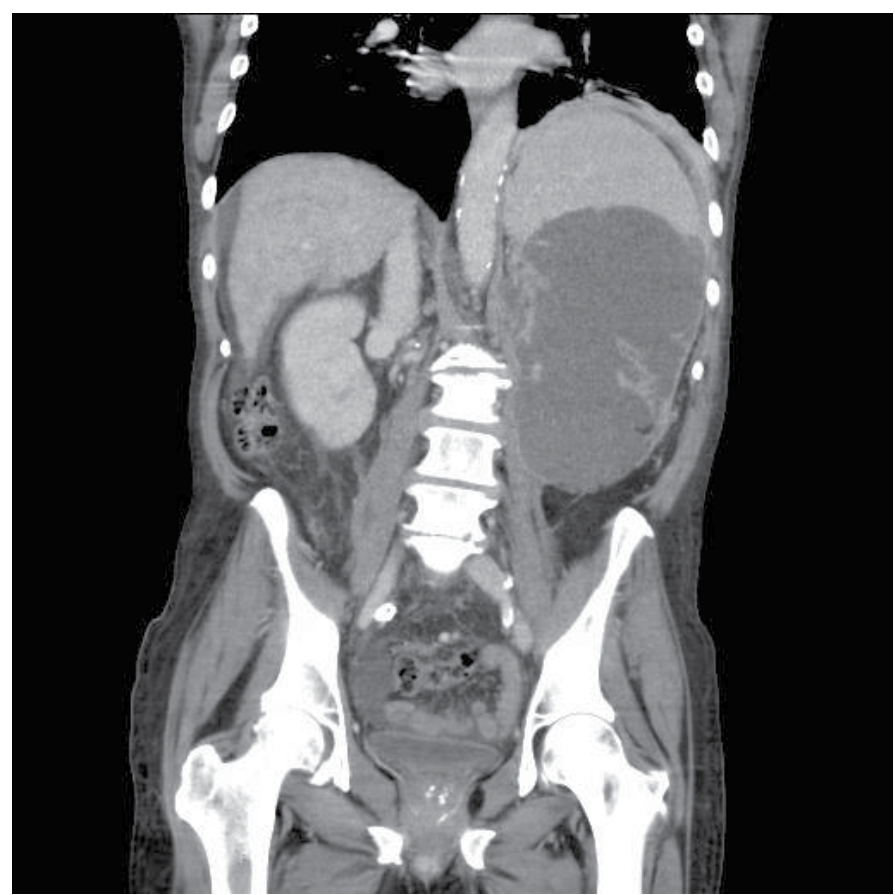

Fig 1b. A computed tomography scan (coronal) on initial presentation. 


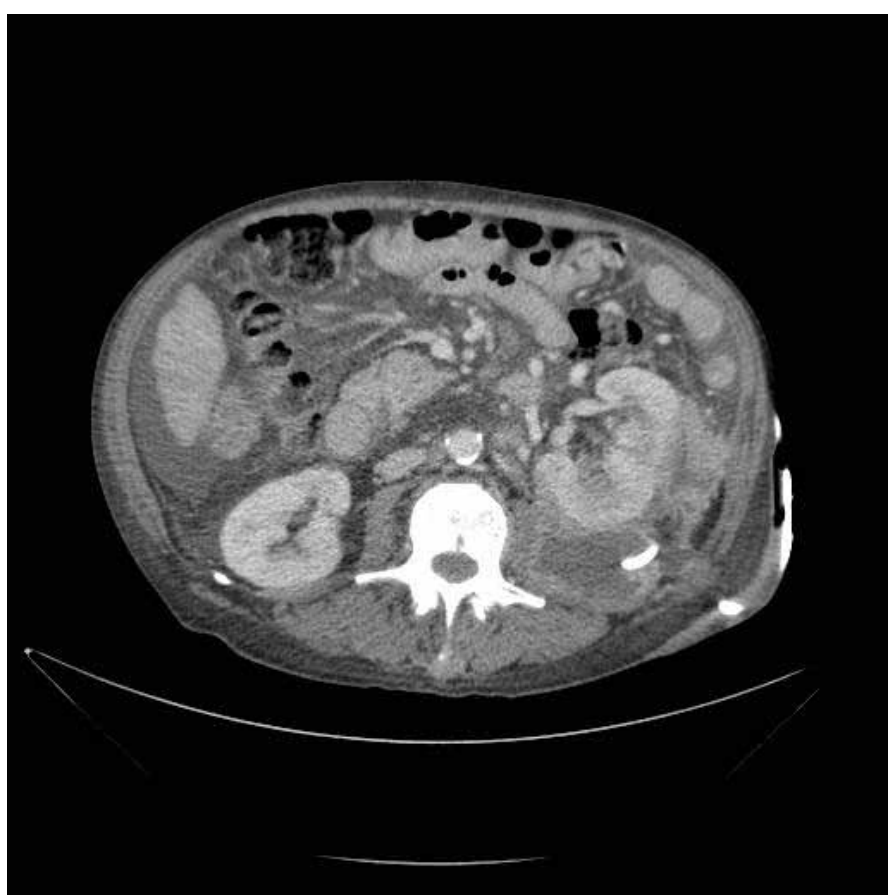

Fig 2. Six days after the placement of the percutaneous drain.

Complications of ureteroscopy range from bleeding to ureteral avulsion. ${ }^{1}$ Our patient conceivably developed a perinephric abscess from a micro-perforation, leading to extravasation of urine or irrigant. One retrospective study, looking at 400 rigid ureteroscopies, found minor mucosal injuries in $5.4 \%$ and ureteral perforation with extravasation in $2.7 \%$ of their cohort. ${ }^{2}$
The most common predisposing factors for the development of perinephric abscesses are urinary tract infections and renal calculi. ${ }^{3}$ Patients usually present with fever followed by flank pain. Stents are unnecessary unless there is evidence of renal obstruction or ongoing urine leakage. Percutaneous drainage is an effective therapeutic tool with a success rate of $86.3 \% .{ }^{4}$

University of Pittsburgh Medical Center, Department of Urology, Pittsburgh, PA

Competing interests: None declared.

This paper has been peer-reviewed.

\section{References}

1. Johnson DB, Pearle MS. Complications of ureteroscopy. Urol Clin North Am 2004;31:157-71.

2. Abdelrahim AF, Abdelmaguid A, Abuzeid $H$, et al. Rigid ureteroscopy for ureteral stones: factors associated with intraoperative adverse events. J Endourol 2008;22:277-80.

3. Meng MV, Mario LA, McAninch JW. Current treatment and outcomes of perinephric abscesses. J Urol 2002;168(4P+1):1337-40.

4. Capitán Manión C, Teiido Sánchez A, Piedra Lara JD, et al. Retroperitoneal Abscesses. Scand J Urol Nephrol 2003;37:139-44.

Correspondence: Dr. Bishoy Gayed, Department of Urologic Surgery, University of Pittsburgh Medical Center, 3471 5th Ave., Suite 700, Pittsburgh, PA 15213; gayedb@gmail.com 